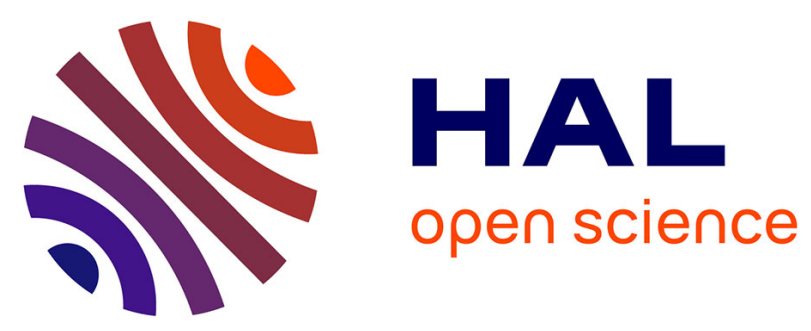

\title{
From MEMS to NEMS: closed-loop actuation of resonant beams beyond the critical Duffing amplitude
}

Jérôme Juillard, Bonnoit Alain, Emilie Avignon, S. Hentz, Kacem N, Eric Colinet

\section{- To cite this version:}

Jérôme Juillard, Bonnoit Alain, Emilie Avignon, S. Hentz, Kacem N, et al.. From MEMS to NEMS: closed-loop actuation of resonant beams beyond the critical Duffing amplitude. IEEE Sensors, Oct 2008, Lecce, Italy. pp. 510-513, 10.1109/ICSENS.2008.4716489 . hal-00351209

HAL Id: hal-00351209

https://hal-centralesupelec.archives-ouvertes.fr/hal-00351209

Submitted on 8 Jan 2009

HAL is a multi-disciplinary open access archive for the deposit and dissemination of scientific research documents, whether they are published or not. The documents may come from teaching and research institutions in France or abroad, or from public or private research centers.
L'archive ouverte pluridisciplinaire HAL, est destinée au dépôt et à la diffusion de documents scientifiques de niveau recherche, publiés ou non, émanant des établissements d'enseignement et de recherche français ou étrangers, des laboratoires publics ou privés. 


\section{From MEMS to NEMS: closed-loop actuation of resonant beams beyond the critical Duffing amplitude}

\author{
J. Juillard, A. Bonnoit, E. Avignon \\ SSE \\ SUPELEC \\ Gif-sur-Yvette, France \\ jerome.juillard@supelec.fr
}

\author{
S. Hentz, N. Kacem, E. Colinet \\ CEA-LETI \\ MINATEC \\ Grenoble, France \\ sebastien.hentz@cea.fr
}

\begin{abstract}
Because of its moderate cost in terms of electronics, resonant sensing has become commonplace in the context of MEMS and NEMS devices. It is usual to drive such resonators below the critical open-loop Duffing amplitude, above which the oscillations become unstable. However, when scaling sensors down to NEMS, nonlinearities may occur at very low amplitudes, making oscillations very difficult to detect. This paper describes a very general way to compute the critical amplitude in open-loop operation for beam resonators, before it focuses on closed-loop Duffing-type resonators. The major contribution of this paper is the use of describing function analysis validated by numerical simulations to show that it is possible to obtain stable oscillations with amplitudes much larger than the critical Duffing amplitude. As a practical consequence, the measured currents are significantly increased and the constraints on the sensing electronics can be relaxed.
\end{abstract}

\section{INTRODUCTION}

Resonant sensing consists in measuring the frequency shift of a system subject to the variation of a given physical quantity. Because of its moderate complexity, this measurement technique is becoming commonplace in the context of MEMS and NEMS devices [1]. This paper focuses on closed-loop resonant sensors, where the micromechanical structure, which can usually be modelled as a Duffing pendulum [2], is brought to oscillate by being placed inside a nonlinear feedback loop. The motion of the electrostatically-actuated Euler-Bernoulli beam (Fig. 1-2) is described by the usual non-linear partial differential equation of the fourth order in space:

$$
\frac{E b h^{3}}{12} \frac{\partial^{4} w}{\partial x^{4}}-T(w) \frac{\partial^{2} w}{\partial x^{2}}+\mu \frac{\partial w}{\partial t}+\rho b h \frac{\partial^{2} w}{\partial t^{2}}=f_{\text {elec }},
$$

where $w$ are the displacements of the beam, $E$ is Young's modulus, $\rho$ is the material's density, $\mu$ is a damping factor, $T$ is an axial force caused by the beam elongation and $f_{\text {elec }}$ is the electrostatic force density.

Assuming $w=a(t) w_{0}(x)$, where $w_{0}(x)$ is the first linear mode shape with eigenvalue $\lambda_{0}{ }^{4}$, the Galerkin procedure can be used to describe the response of the beam. This yields:

$\omega_{0}^{2} a+\frac{\omega_{0}}{Q} \dot{a}+\ddot{a}=\frac{1}{\rho b h} \int_{0}^{L} f_{\text {elec }} w_{0} d x-\alpha a^{3}$,

where $\omega_{0}{ }^{2}=\frac{E h^{2} \lambda_{0}{ }^{4}}{12 \rho}$ and $\alpha=\frac{E}{2 \rho L}\left(\int_{0}^{L}\left(\frac{\partial w_{0}}{\partial x}\right)^{2} d x\right)^{2}$.

The equivalent block-diagram of (2) is represented in Fig. 3.

In the first part of this paper, we show how describing function analysis (DFA) [3] can be used to account for the critical amplitude phenomenon (Fig. 3) occurring in Duffing oscillators [4]. It is found that, in the limit of large quality factors, the amplitude at the critical point is $w_{\text {crit }} \approx 1.463 h Q^{-1 / 2}$ and the amplitude of the peak of the frequency response is $w_{\max } \approx 1.685 h Q^{-1 / 2}$. For large quality factors, this sets an upper limit to the signal amplitudes that can be measured at the output of an open-loop Duffing oscillator without incurring hysteretic behaviour or dynamic instability and, as a consequence, is detrimental to the sensor resolution.

In the second part of the paper, the closed-loop problem, in which the excitation is a nonlinear function of the system's state, is studied. We show how DFA applies in this case. The analytical expression of the oscillation amplitude and frequency of a pulse-actuated microbeam (for which a short negative or positive voltage pulse is applied every time 


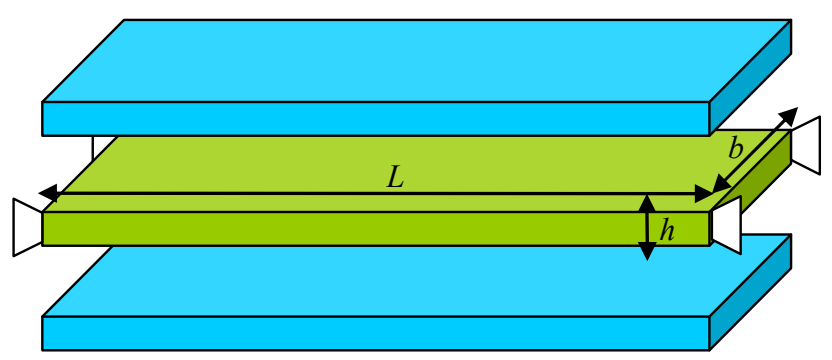

Figure 1. Electrode (blue)-beam (green) arrangement and notations (top and bottom gaps are identical). The clamped-clamped microbeam is the mobile midpoint of a capacitive half-bridge (Fig. 2).

the measured signal crosses zero [5]) is obtained, showing that there is only one possible oscillation state for such a system and that it is stable. Finally, these analytical results are compared with simulations of the fully nonlinear system, in the practical case of the micro-accelerometer developed in the ANR-funded M\&NEMS project.

\section{CAlCUlation OF THE CRITICAL DUfFING AMPLITUDE}

To determine the critical Duffing amplitude, we study a simpler problem where electrostatic nonlinearities are neglected. Let us then consider a sinusoidally-forced Duffing pendulum:

$\omega_{0}^{2} a+\frac{\omega_{0}}{Q} \dot{a}+\ddot{a}=f \sin (\omega t)-\alpha a^{3}$.

The transient problem may be studied with a method of averaging, along the lines developed in [4]. In the present case, however, it is sufficient to use DFA to establish the steady-state properties of (3). Under the assumptions that the linear part of the system filters out the harmonics introduced by the nonlinear part and that the system has reached a periodic regime, we have:

$a=A \sin (\omega t+\varphi)$.

The expressions of $\dot{a}$ and $\ddot{a}$ can be derived from (4) in a straightforward fashion. Neglecting higher-order harmonics and switching to complex notations, the signal at the output of the nonlinear part (Fig. 4) is:

$$
N(j \omega)=\frac{3}{4} \alpha|A|^{2} A(j \omega)
$$

Using classical system theory, one can then write:

$$
\frac{A(j \omega)}{F(j \omega)}=\frac{1}{\left(U(\omega)+\frac{3}{4} \alpha|A|^{2}\right)+j V(\omega)}
$$

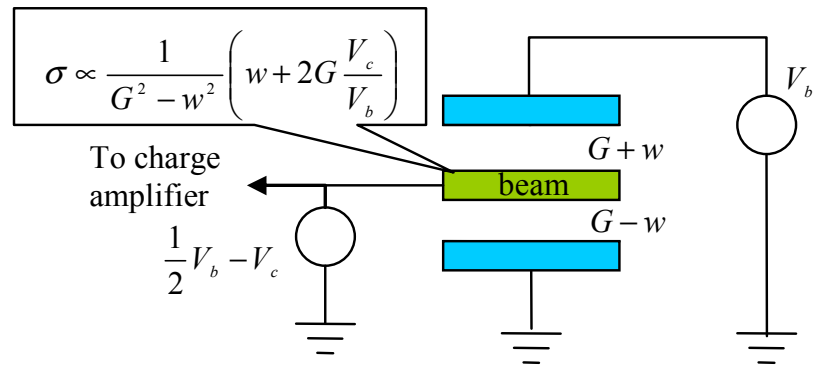

Figure 2. Cross-section of the electrode/beam arrangement of Fig. 1 and notations. The mobile beam is used for sensing and for actuating. $V_{c}$ should be properly chosen, to measure the beam displacements without crosstalk.

where $U=\omega_{0}{ }^{2}-\omega^{2}$ and $V=\omega \omega_{0} / Q$. Taking the square of the modulus of (6) and expanding yields:

$A^{6}+\frac{8}{3 \alpha} U A^{4}+\frac{16}{9 \alpha^{2}}\left(U^{2}+V^{2}\right) A^{2}-\frac{16}{9 \alpha^{2}} f^{2}=0$.

Since $A$ is positive, (7) may have up to three distinct real roots. The critical regime is reached when (7) has one triple real root. In this case, (7) has the form $\left(A^{2}-C\right)^{3}=0$, with positive $C$. Thus, we find that:

$C=-\frac{8}{9 \alpha} U$ and $C^{2}=\frac{16}{27 \alpha^{2}}\left(U^{2}+V^{2}\right)$.

Eliminating $C$ from (8) leads to a second-order equation in $\omega^{2}$. It is then simple to derive the expression of the pulsation of the critical point:

$\omega_{\text {crit }}=\omega_{0} \sqrt{1+\frac{3}{4 Q^{2}}}+\operatorname{sign}(\alpha) \frac{\sqrt{3}}{2} \frac{\omega_{0}}{Q}$.

In the limit of large quality factors, this becomes:

$\omega_{c r i t}=\omega_{0}+\operatorname{sign}(\alpha) \frac{\sqrt{3}}{2} \frac{\omega_{0}}{Q}$

One can then use the expression of the beam's central frequency and stiffening coefficient to obtain the corresponding critical displacement amplitude:

$w_{\text {crit }} \approx 1.463 h Q^{-1 / 2}$

At the same excitation amplitude, the maximum displacement (corresponding to the maximum of the system frequency response) is found equal to $w_{\max } \approx 1.685 h Q^{-1 / 2}$. 


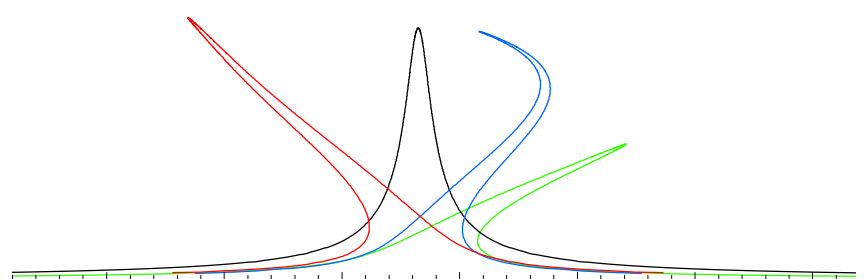

Figure 3. Possible frequency responses of an electrostatic MEMS device, for different bias voltages (from [4]): softening behaviour (red curve), linear behaviour (black curve), hardening behaviour (green curve) and mixed behaviour (blue curve).

\section{STUDY OF THE CLOSED-LOOP PROBLEM}

\section{A. Describing function analysis of closed-loop systems}

With the same assumptions as in section II, the selfoscillating closed-loop nonlinear system of Fig. 5 may be analysed with DFA: its amplitude and frequency can be determined, as well as the stability of the oscillations.

In order to use DFA on a closed-loop system, one must:

1. assume $a=A \sin (\omega t)$,

2. find the equivalent complex gain $N(A, \omega)$ of the nonlinear part $F(a, \dot{a})$,

$N(A, \omega)=\frac{\omega}{2 \pi A} \int_{0}^{2 \pi / \omega} F(a, \dot{a}) \exp (j \omega t) d t$.

3. check for an oscillatory regime by verifying the Barkhausen conditions, i.e. solve

$N(A, \omega) H(j \omega)=1$,

for $A$ and $\omega$, where $H(j \omega)$ is the transfer function of the linear part.

Steps 1 and 2 are almost equivalent to the approach presented in section II. Step 3 is equivalent to saying that the total phase lag in the loop is equal to zero.

\section{B. DFA of the pulse-actuated microbeam}

In the case of the electrostatic microbeam shown in Fig. 1-2, one may write:

$f_{\text {elec }}=\frac{2 \varepsilon_{0} b}{\left(G^{2}-w^{2}\right)^{2}}\left(\frac{V_{b}}{2} w-V_{c} G\right)\left(\frac{V_{b}}{2} G-V_{c} w\right)$,

where $V_{c}$ consists in short voltage pulses of amplitude $\pm V_{p}$ and duration $T_{p}$ that are triggered when the detected signal crosses zero [5]. In the present case, the detected signal is the

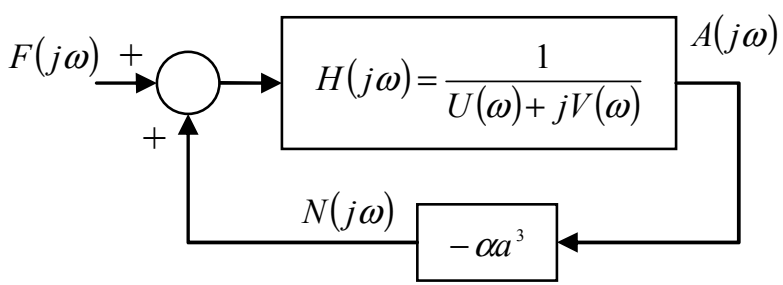

Figure 4. Equivalent block diagram representation of the open-loop Duffing pendulum.

charge accumulated on the microbeam. The charge density is given by:

$$
\sigma \propto \frac{1}{G^{2}-w^{2}}\left(w+2 G \frac{V_{c}}{V_{b}}\right)
$$

It is simple to show that the total charge crosses zero whenever $a$ (and, as a consequence, $w$ ) crosses zero. Thus, assuming the pulses are short (with respect to the period of oscillation), one may neglect the $V_{c} w$ term appearing in (14). We also neglect nonlinear electrostatic softening, i.e. we suppose $\left(G^{2}-w^{2}\right)^{2} \approx G^{4}$. Thus:

$f_{\text {elec }} \approx \frac{\varepsilon_{0} V_{b} b}{G^{3}}\left(\frac{V_{b}}{2} w-V_{c} G\right)$.

Finally, (16) can be injected into (2). This yields:

$\omega_{t o t}{ }^{2} a+\frac{\omega_{0}}{Q} \dot{a}+\ddot{a}=D V_{c}-\alpha a^{3}=F(a, \dot{a})$

where $\omega_{t o t}{ }^{2}=\omega_{0}{ }^{2}-\frac{\varepsilon_{0}}{2 \rho h} \frac{V_{b}{ }^{2}}{G^{3}}$ and $D=\frac{\varepsilon_{0}}{\rho h} \frac{V_{b}}{G^{2}} \int_{0}^{L} w_{0} d x$.

The equivalent gain of $F(a, \dot{a})$ is found to be:

$$
N(A, \omega)=-\frac{3}{4} \alpha A^{2}+j \omega \frac{2 D T_{p}}{\pi A} V_{p}
$$

Using this expression in (13) yields one unique solution:

$$
A_{o s c}=\frac{2 Q}{\pi \omega_{0}} D V_{p} T_{p} \quad \omega_{o s c}=\sqrt{\omega_{t o t}{ }^{2}+\frac{3}{4} \alpha A_{o s c}{ }^{2}}
$$

This clearly shows that there exists only one possible selfoscillation state for the electrostatic pulse-actuated microbeam. 


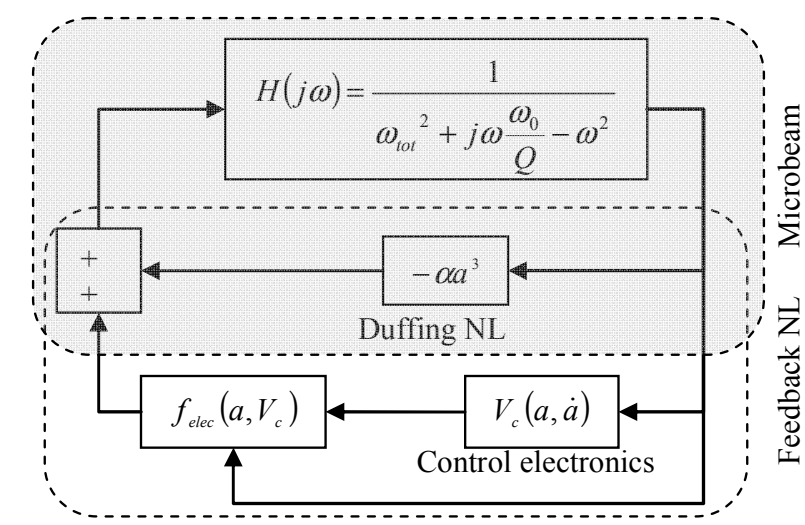

Figure 5. Equivalent block diagram of an electrostatically-actuated, stress-stiffened microbeam (shaded area) with its nonlinear feedback electronics and actuation scheme. The system may be looked at as a linear system with one complex feedback nonlinearity.

The approach described in [3] can be used to determine the stability of the oscillations. The oscillations are stable (in the sense that the system goes back to its original oscillatory state after a slight perturbation) when the following inequality is satisfied:

$\frac{\partial R}{\partial A} \frac{\partial I}{\partial \omega}-\frac{\partial I}{\partial A} \frac{\partial R}{\partial \omega}>0$

where $R$ and $I$ are the real and imaginary part of $N(A, \omega) H(j \omega)$ and the partial derivatives are taken at $A=A_{\text {osc }}$ and $\omega=\omega_{\text {osc }}$. In the present case, it is simple to show that $\alpha>0$ is sufficient for the oscillations to be stable. However, it should be noted that, for very large displacement amplitudes, the electrostatic softening is no longer linear. As a consequence, it is impossible to predict the dynamic pull-in amplitude of the beam with this method. This is the subject of ongoing work.

\section{Simulation AND RESUlTS}

In this section, we compare the results obtained by simulation of (2) to the analytical expressions obtained in section III. The parameters of the resonator correspond to those of the accelerometer structure developed in the ANRfunded M\&NEMS project: $L=25 \mu \mathrm{m}, \quad b=500 \mathrm{~nm}$, $h=G=250 \mathrm{~nm} \quad\left(\omega_{0}=2.2 \times 10^{7} \mathrm{rad}_{\mathrm{s}}^{-1}.\right), \quad Q=6000 \quad$ and $V_{b}=1.2 \mathrm{~V}$. The duration of the impulses is set to $T_{p}=10 \mathrm{~ns}$. The results obtained with Matlab-Simulink for different values of $V_{p}$ are shown in Fig. 6 and compared to those derived from the analytical expressions of (19). There is a very good agreement between the two approaches even for very large displacement amplitudes: this validates the assumptions made in section III, in particular those concerning the neglected terms in (14). The results show that it is not only possible to actuate the beam with amplitudes much larger than the critical Duffing amplitude

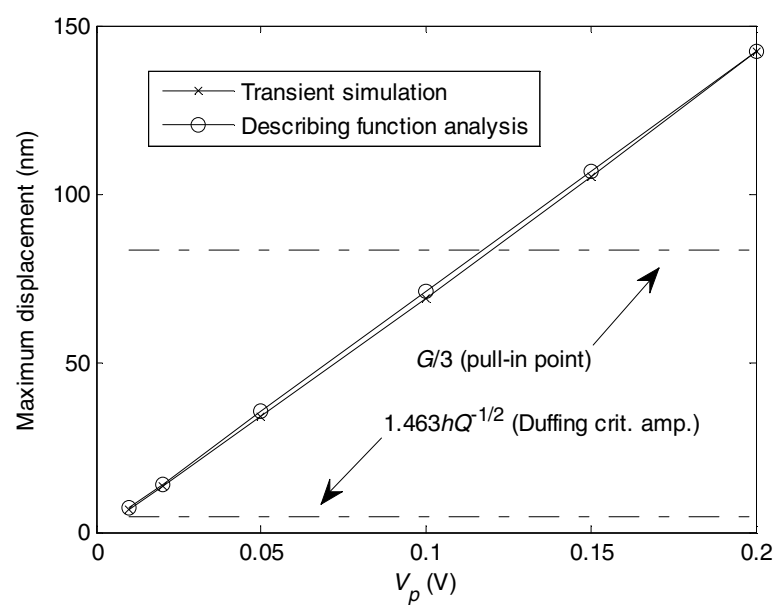

Figure 6. Comparison of maximum displacements obtained by DFA (circles) and by transient simulation (crosses).

( $w_{\text {crit }}=4.7 \mathrm{~nm}$ ), but also to have stable oscillations beyond the static pull-in point $\left(G / 3 \approx 83 \mathrm{~nm}\right.$ ). For $V_{p}=0.2 \mathrm{~V}$, the current that must be amplified by the analog front-end is about 50 times larger than the current corresponding to the critical amplitude. For larger values of the pulse amplitude, dynamic pull-in is observed in simulation. The analytical model should be improved to account for this phenomenon (the approach presented in [4] could be used, for example).

\section{CONCLUSION}

We have shown how DFA can be used to determine the oscillatory regime of a Duffing resonator, operated in open or closed-loop. In particular, we have calculated the critical Duffing amplitude for a clamped-clamped beam and shown this to be equal to $w_{\text {crit }} \approx 1.463 h Q^{-1 / 2}$. Furthermore, we have shown that a pulse-actuated electrostatic microbeam can be actuated with amplitudes much larger than this critical value, without risk of hysteretic behaviour. As a practical consequence, the measured currents are significantly increased and the constraints on the sensing electronics can be relaxed. This was validated by comparing the analytical results given by DFA to those obtained with transient simulations of the resonant accelerometer sensing cell developed in the framework of the M\&NEMS project.

\section{REFERENCES}

[1] T. Roessig, "Integrated MEMS tuning fork oscillators for sensor applications," Ph.D. dissertation, Department of Mechanical Engineering, University of California, Berkeley, 1998

[2] V. Kaajakari, T. Mattila, A. Oja, H. Seppä, "Nonlinear limits for single-crystal silicon microresonators", J. MEMS, vol 13, No 5, Oct 2004

[3] A. Gelb, W. Van der Velde, "Multiple-input describing functions and nonlinear system design", McGraw-Hill, New-York, 1968

[4] N. Kacem, S. Hentz et al, "From MEMS to NEMS: modelling and characterization of the non linear dynamics of resonators, a way to enhance the dynamic range", Proceedings of Nanotech 2008, Boston

[5] E. Colinet, J. Juillard, S. Guessab, R. Kielbasa., "Actuation of resonant MEMS using short pulsed forces", Sensors and Actuators A, $\mathrm{n}^{\circ} 115,2004$ 